\title{
APPARATUS FOR RECORDING CONTINUOUS DISCRIMINATION REACTIONS
}

\author{
BY H. C. McCOMAS
}

Princeton University

A series of discrimination reactions has been used in connection with a number of investigations having widely divergent interests. The records obtainable in time and accuracy for such reactions afford side lights upon a variety of processes. It is not surprising, therefore, to find some type of discrimination apparatus, from Seashore's "Psychergograph" of fifteen years ago to the numerous forms of today, employed in experiments on mental efficiency, concentration of attention, influence of distractions, motor impulses, etc.

The devices used are adapted, of course, to the immediate purposes of each experimenter, though the general principle is the same; namely, to present several different stimuli and to record the several possible reactions continuously. To obtain reliable results in this work a number of conditions must be squarely met. That part of the apparatus with which the subject has to do must be noiseless, with no moving parts that afford distractions. The stimuli must be constant in intensity and sufficiently varied in the order of presentation to obviate anticipation. The type of response must admit sufficient variation to preclude the quick formation of habit. Fatigue in sense-organ and reacting muscles must be reduced to a minimum. Time records should be obtainable for both right and wrong reactions during variable periods.

In addition to these indispensable requirements it is desirable to have an apparatus to which all subjects become quickly adapted. This shortens the practice curves. If the reaction is simple and the discrimination is not complicated, the training period is reduced; and the results of different subjects are more comparable. For a large class of subjects it is a decided advantage to have work which is interesting. The kymograph 
records are long and should be easily legible. This can be partially accomplished by markers with wide amplitude and set close together. As this type of apparatus is not manufactured it is desirable that it should be easily and cheaply made.

The apparatus to be described meets these requirements more fully than the usual types. It is capable of many adaptations. The work in which it has been most used was in obtaining ten minute records of continuous discriminations. The procedure was briefly this: the subject sat in one room, with the exposure screen and reacting keys; the experimenter and the rest of the apparatus were in another room. The subject reacted to four differently colored lights, by pressing different keys. He learned which keys extinguished which lights before the records began, and then he reacted as quickly as he could with the fewest possible false reactions. The instant one colored light was extinguished another appeared; the order of their appearance was miscellaneous. The subject therefore controlled the rate of the exposures. The records were read for ten second intervals and showed that the average subject makes from eight to thirteen correct reactions in such an interval and from no false reactions to three or four. The time of reaction and the number of false reactions increase as the test goes on. The records show typical differences in subjects and characteristic forms of reaction for some subjects at different times of the day.

In describing the apparatus it may be conveniently considered in three parts: first, the stimulus and reaction table; second, the automatic switchboard; third, the recording instruments.

Upon a small table is placed a green cardboard screen with a small ground glass window in its center. In a rack behind the window are four Mazda, six-volt bulbs. In front of these are four different colored gelatine strips. The bulbs are connected with a Hyray battery. This service brings the filaments to incandescence in about $.05 \mathrm{sec}$. after a circuit is closed. Four telegraph sending keys are placed in front of the screen upon the table, and in such a position that they 
are easily seen in indirect vision when the eyes are fixated upon the window (Plate I.). They are wired to make two circuits; one by the contacts at the far end of the rocking arm and the other by the contacts at the near end (Plate IV., $L C$ and $M C$ ). The reaction movement breaks the circuit in which the light is made and at the same time it makes a circuit which actuates a marker on the kymograph. Thus

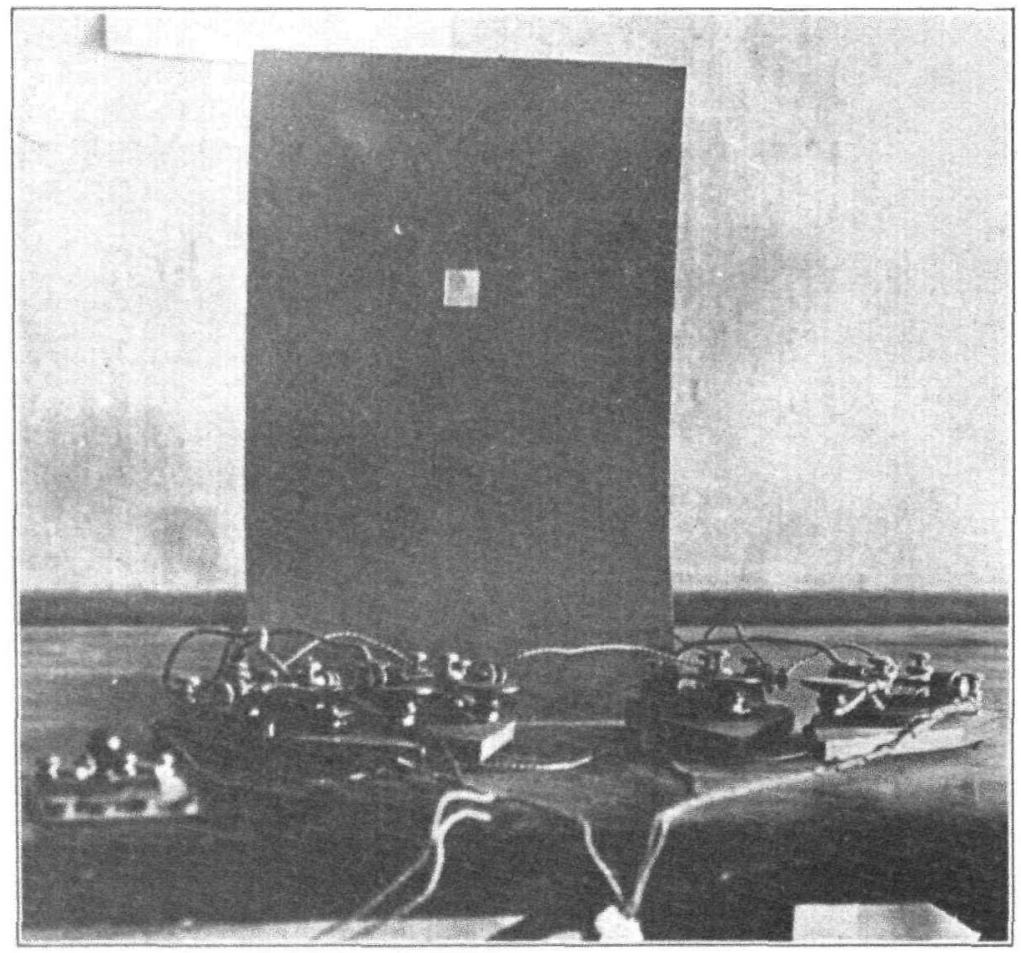

PLATE I.

the light is extinguished and a reaction recorded by one movement.

An automatic switchboard is used to remake the light circuits. This device is easily and cheaply made by fastening a 'secondary' electric clock opposite a board on which there is a circle of radiating copper contacts (Plate II.). The 'secondary' is a simple mechanism by which the minute 
hand of the clock is moved forward with each break of the current running into the magnet. By placing a trailer contact on the minute hand and adjusting it to pass over the radiating copper contacts, it is possible to have the hand move forward to a new contact and make a new circuit whenever a circuit is broken by a reaction. The sixty copper contacts are fastened upon the supporting board as radii coincident with the minute divisions of a clock face. From the contacts wires pass through the board and rest in four bowls of mercury, fifteen wires to the bowl. From each bowl a wire runs to

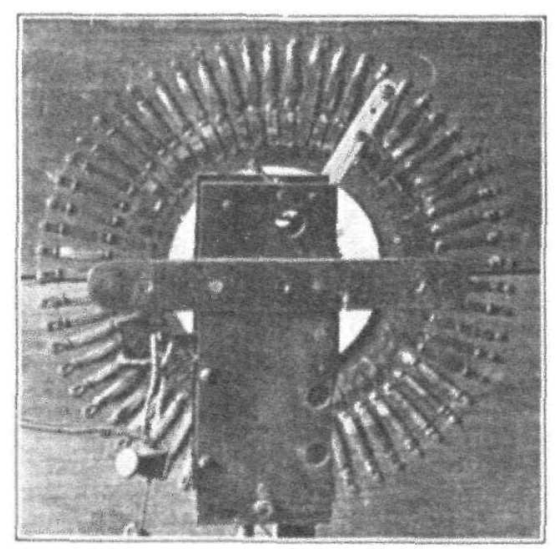

Plate II.

each of the four keys. When a light circuit is broken at one of the reaction keys, such as $L C$ in key $K_{4}$, Plate IV., the light $L_{4}$ is extinguished, the current ceases to pass through the coils in magnet $M$, this releases armature $A$ and the arm $A R$ swings counter clockwise moving the hand $H$ forward to another contact. The time required to pass from one contact to the next is $.08 \mathrm{sec}$. This can be shortened by placing the copper strips closer together, and by adding to the weight at $N$.

The records are taken upon a kymograph. A Jaquet marker gives the time in seconds. A marker in circuit with the armature of the clock registers each correct reaction and a marker in circuit with the reacting keys scores all the reactions 
made. These markers are constructed from two telegraph sounders with long pliable copper strips to give a wide amplitude (Plate III.). They are set close together, the all-reactions registering immediately below the correct reactions.

The wiring of these three divisions of the apparatus can be easily understood by reference to Plate IV. There are two independent battery circuits, the light circuit and the marker circuits. The light circuit may be traced from the battery $B_{1}$ to the four bulbs $L_{1} L_{2} L_{3} L_{4}$ and from these to the four reacting keys $K_{1} K_{2} K_{3} K_{4}$. If all the circuits stood open except $K_{4}$, the current would pass through $L C$ in to the wire $R_{4}$ running to the automatic switchboard. There it would enter

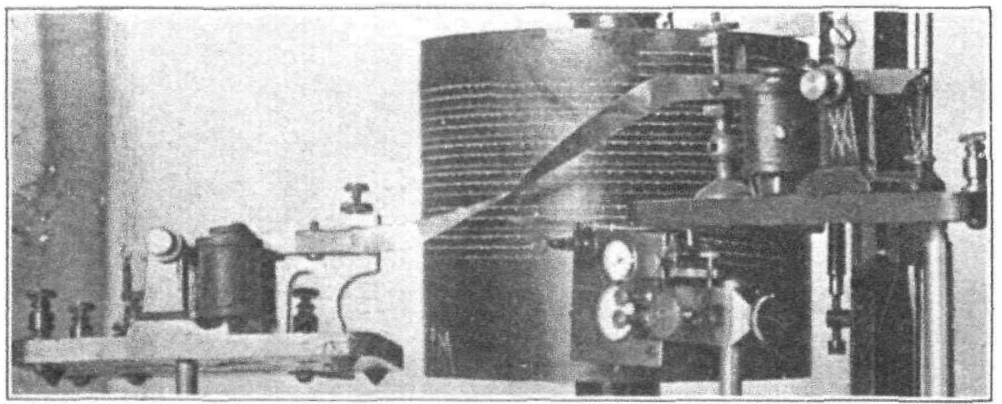

Plate III.

one of the four mercury bowls from which it would pass to the radiating copper strips $C S$. On one of these the trailer contact $H$ is resting. The current, therefore, passes through $H$ down to the magnet $M$, actuating armature $A$ and thence back to the battery.

The other two circuits lead from the battery $B_{2}$, they are the marker circuits. With the reaction to each light the current in $M$ is broken and the lever $A R$ moves about the axle $P$. With each movement a contact is made at $N$ and the marker $Y$ registers on the kymograph $K Y$ giving a record of the right reactions. The marker $Z$ is actuated by closing such a contact as $M C$ in $K_{4}$. The wires $F_{1}$ and $F_{2}$ connect the keys with the battery and the marker.

If it is desired to give a longer series of discrimination 
reactions than sixty at a sitting it may be advisab!e to put a sliding switch in $R_{1} R_{2} R_{3} R_{4}$ and thus shift the order in which the lights appear in $L_{1} L_{2} L_{3} L_{4}$. However, many subjects do

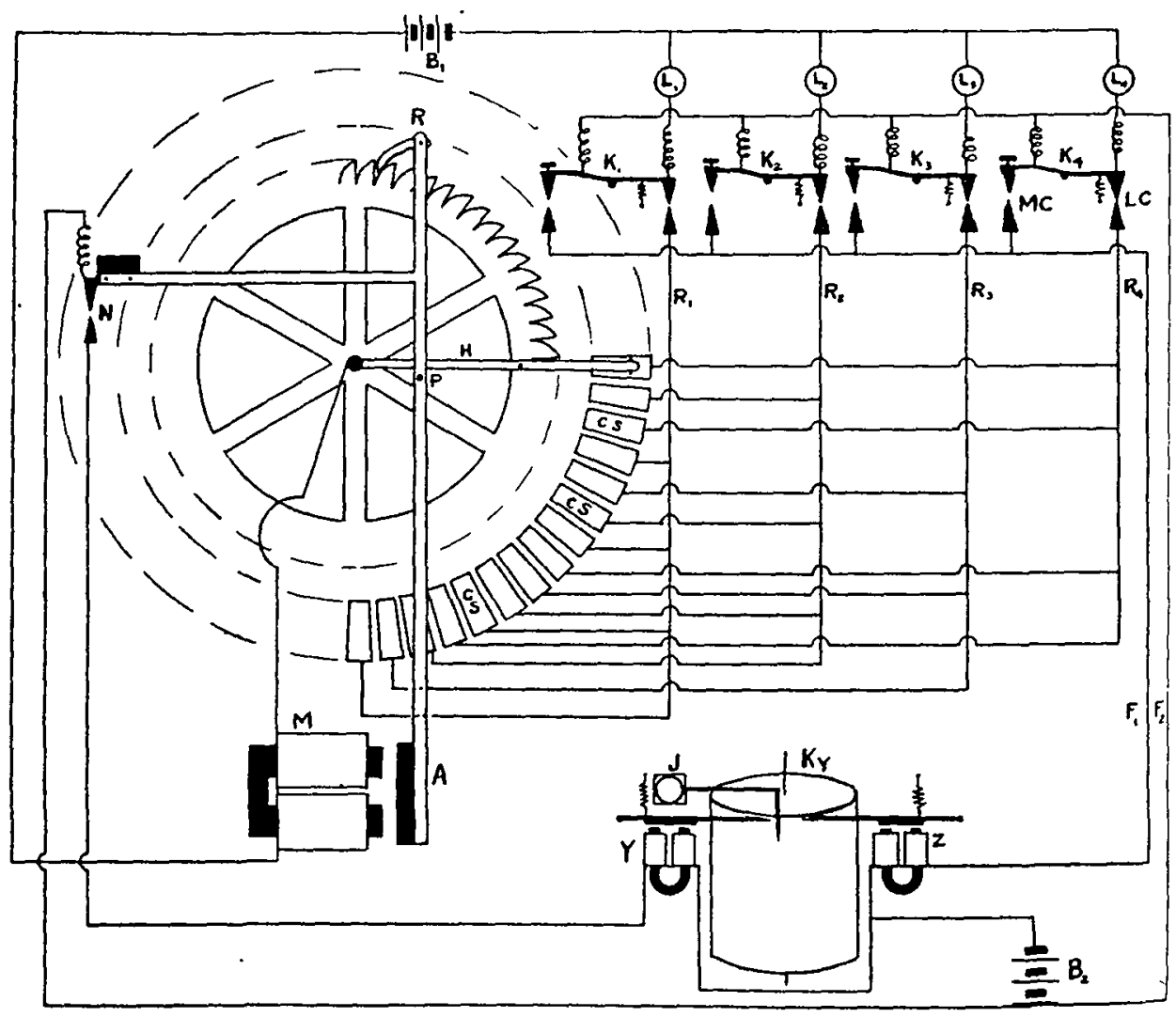

Plate IV.

$L_{1} L_{2} L_{3} L_{\text {A }}$, lamps; $K_{1} K_{2} K_{3} K_{4}$, reaction keys; $L C$, contacts for lighting circuit; $M C$, contacts for marker circuit; $R_{1} R_{2} R_{3} R_{4}$, connections with automatic switchboard; $F_{1} F_{2}$, connections with marker; $C S$, copper strips on clock; $H$, clock hand connection; $M$, magnet; $A$, armature; $R$, ratchet engaging wheel; $P$, axle suspending arm, $R-A$; $N$, contacts; $K Y$, kymograph; $J$, Jaquet time marker; $Y$, marker of right reactions; $Z$, marker of all reactions; $B_{1} B_{2}$, batteries.

not learn the order or any part of it, though it is repeated ten times in ten minutes.

The above arrangement of exposure and recording apparatus gives a very serviceable device for discrimination work. There 
are no distractions. The eye may remain fixed on the stimuli. The reactions are simple and not fatiguing. The order of. presentation is not repeated for sixty exposures and the right and wrong reactions are both recorded. Not the least attractive feature is the interest the reactions arouse in the subject, who willingly struggles with a long series "for the fun of it." 